\title{
Efecto de Geophila macropoda (Rubiaceae) como arvense de cobertura en la erosión hídrica en bananales de Guápiles, Limón, Costa Rica
}

\author{
Jimmy Murillo, Víctor Hugo Méndez-Estrada \& Steven Brenes Prendas \\ jmv_jimmy@yahoo.es; vmendez@uned.ac.cr; sbrenesp@gmail.com
}

Recibido 20-II-2016 • Corregido 04-IV-2016 • Aceptado 17-VII-2016

\begin{abstract}
Effect of Geophila macropoda (Rubiaceae) as "weed cover" on hydric erosion in banana plantations, Guápiles , Limón , Costa Rica. The establishment of banana companies in Costa Rica brought employment and infrastructure improvements, but also social problems, destruction of forests, soil erosion and pollution of rivers and streams. Erosion in banana plantations may be diminished with plant cover. Here we analyze the effect of the plant Geophila macropoda on erosion caused by water in Guápiles, Costa Rica. We chose three soil classes I, II and III with and without G. macropoda. Between June and August 2012 periodic measurements were performed once a week, covering periods of low, medium and high rainfall. We found that erosion increases with precipitation and that cover with this plant reduces erosion in all soil types.
\end{abstract}

Key words: runoff, erosion, weeds, crop, rain, sediments.
RESUMEN: El establecimiento de compañías bananeras en Costa Rica trajo fuentes de empleo y mejoras infraestructura, pero también problemas sociales, destrucción de bosques, erosión del suelo y contaminación de ríos y quebradas. La erosión en bananales puede verse disminuida con prácticas de cobertura vegetal. Aquí analizamos el efecto de la planta arvense Geophila macropoda (oreja de ratón) en la erosión en Guápiles, Costa Rica. Elegimos tres clases de suelos I, II y III cubiertos con oreja de ratón y sin ella. Entre junio y agosto de 2012 se realizaron mediciones periódicas una vez por semana, abarcando periodos de baja, media y alta precipitación. Encontramos que la erosión aumenta con la precipitación y que en todos los suelos la cobertura de G. macropoda redujo la erosión.

Palabras clave: escorrentía, erosión, malas hierbas, cultivo, lluvia, sedimentos.
La introducción del banano en Costa Rica se dio en 1871 y desde entonces se ha expandido hasta abarcar 43031 ha (Kruiter, 1989; Villalobos, 2006). La producción de banano se realiza principalmente por empresas transnacionales que se concentran en Sarapiquí (Heredia) y desde el cantón de Pococí hasta Sixaola, Talamanca, Limón [Corporación Bananera Nacional (CORBANA), 2011a].

Las compañías bananeras trajeron a Costa Rica desarrollo, infraestructura; mejoras en las vías públicas, viviendas y escuelas; fuentes de empleo, áreas de recreación, fundaciones sociales, zonas de conservación e ingresos por impuestos de venta del producto en (Salguero, 2007; Yepes, 2012). Sin embargo han provocado daños al ambiente por medio de la destrucción de los bosques, erosión del suelo, contaminación de fuentes de agua con los subproductos del banano y uso de pesticidas (Morgan, 1997; Núñez, 2001; Chacón, 2003).

La actividad bananera deja el suelo descubierto y expuesto a la erosión hídrica, el agua fácilmente desprende sus partículas finas, con residuos químicos, que transporta y sedimenta en los canales de desagüe pluviales propios de la plantación bananera (Hudson, 1982; Soto, 2009; Alvarado et al., 2014), lo que provoca la disminución del rendimiento agrícola en el cultivo del banano [Reduciendo el Escurrimiento de Plaguicidas al Mar Caribe-(RepCar), 2000; Moreno, Blanco \& Mendoza, 2009]. Esas pérdidas por erosión se ven influenciadas por la textura del suelo, los de textura gruesa cuentan con alta capacidad de absorción del agua superficial, por lo que presentan menos erosión. Los suelos arcillosos son de poca infiltración, lo que propicia el arrastre de las partículas por el agua y su degradación es mayor (Morgan, 
1997); por lo tanto, el mapeo por clases de suelos es importante en las prácticas agrícolas.

Un método para contrarrestar las malezas en el cultivo de banano es la utilización de arvenses: coberturas vegetales que no compitan con el cultivo (Martínez, 1998), pero su inadecuada elección podría ocasionar que se vuelvan competencia del cultivo y que se conviertan en hospederas de plagas que afecten la plantación y lo vuelvan difícil de controlar (Pound, 2003; Rojas, Bermúdez \& Jiménez, 2006; Waele et al., 2006).

Entre los arvenses más usados está Geophila macropoda (Oreja de Ratón, Rubiaceae) (Brenes, 2010; Ramos et al., 2011); se elige porque es rastrera, crece entre los cinco y diez centímetros, prefiere climas frescos o bajo sombra, soporta el paso de trabajadores, requiere de poca agua en las épocas de verano; tallo terrestre, un sistema radical poco profundo y poco difuso; es perenne, se propaga por semilla y vegetativamente [Instituto Interamericano de Ciencias Agrícolas (IICA), 1989; Pinilla \& García, 2002; Vargas \& Abdelnour, 2010; Ramos et al., 2011]. Sus plantaciones disminuyen la pérdida de suelo por erosión hídrica y eólica, disminuyen la temperatura del suelo, albergan enemigos naturales de algunas plagas, aumentan la biodiversidad de los agroecosistemas y eliminan malezas, por lo tanto, la convierte en una cobertura amigable con la plantación de banano (Vargas \& Abdelnour, 2010). Además, muchos arvenses son utilizados como materias primas, medicinales y como indicadores de calidad de sitio y retienen humedad en el suelo (Yglesias, 1997; Díaz et al., 1999; Arias, 2007; Brenes, 2010).

Para el caso de G. macropoda no se han realizado estudios sobre el efecto que tiene su uso como arvense en la disminución de la erosión hídrica según la clasificación del suelo bananero; por lo tanto, se plantea el objetivo de analizar el efecto que tiene su uso como arvense en la disminución de la erosión hídrica según la clase de suelo y los beneficios económicos que se obtienen en el cultivo del banano en una finca bananera en Guápiles, Pococí.

\section{MÉTODOS}

La finca se encuentra en Pococí, Limón, a $12 \mathrm{~km}$ al norte del distrito central de Guápiles; coordenadas 5'56", 5'59" Este-Oeste y 2'52", 2'56" Norte-Sur. Cuenta con 339,11 ha; 317,93 dedicadas al cultivo y producción de banano Cavendish Gran Enano (Samuels \& Segura, 2010). La precipitación está entre los $3500 \mathrm{~mm}$ y los $4000 \mathrm{~mm}$ al año (Soto, 2008) y una temperatura promedio de $26^{\circ} \mathrm{C}$.

La finca tiene 60ha cubiertas con G. macropoda (oreja de ratón), mientras que las restantes 257,93 ha se encuentran sin cobertura y, en ellas se utiliza el control químico para eliminar las malezas.

Escogimos seis puntos de muestreo, ubicados en áreas cercanas unas de otras para evitar que las condiciones climáticas cambiaran (Kruiter, 1989) y que se presentara una inclinación del suelo del 2,8\% (Araya, 2014). Uno en cada clase de suelo, I, II y III, con cobertura entre el $67 \%$ y $100 \%$ de oreja de ratón (G. macropoda) y tres puntos en los mismos tipos de suelo pero sin cobertura (cuadro 1).

CUADRO 1

Áreas de estudio según clase de suelo con y sin coberturas

\begin{tabular}{clc} 
Clase de Suelo & \multicolumn{1}{c}{ Característica } & Área $\left(\mathrm{m}^{2}\right)$ \\
I & Con Cobertura & 288 \\
II & Sin Cobertura & 306 \\
& Con Cobertura & 190 \\
III & Sin Cobertura & 390 \\
& Con Cobertura & 360 \\
& Sin Cobertura & 378 \\
\hline
\end{tabular}

En cada uno de los seis puntos de muestreo se colocó un balde plástico (trampas) con capacidad de diez litros con el fin de capturar las partículas de suelo erosionadas y así medir el nivel de erosión del suelo (Somarriba, 2005). Las trampas se colocaron de acuerdo con la dirección de la escorrentía del agua pluvial en cada sitio y fueron enterradas en el suelo, dejando el borde superior descubierto al mismo nivel del suelo, para que todas las partículas de suelo que se desplacen durante la lluvia queden atrapadas en ellos (Vallejos \& Velázquez, 1998).

El suelo recogido en las trampas fue llevado a un laboratorio y fue secado en un horno durante 24 horas para obtener el peso real en seco del sedimento recolectado (Laprade 2012a, inf. pers), lo que permitió comparar la cantidad de sedimento entre las áreas con cobertura vegetal y sin ella.

El volumen $\left(\mathrm{g} / \mathrm{m}^{2}\right)$ de sedimentos recolectado en cada trampa según el suelo correspondiente, fue convertido de hectáreas a tonelada con la siguiente fórmula:

$$
\frac{x g}{y m^{2}} \times \frac{z K g}{x g} \times \frac{m \text { Ton }}{z K g} \times \frac{y m^{2}}{n \text { ha }}=\text { Ton ha }^{-1}
$$

El nivel de erosión recolectado en las trampas se clasificó en siete grados: natural $\left(<1,0\right.$ Ton ha $\left.{ }^{-1}\right)$, baja $(1,1$ a 3,0 Ton ha $\left.{ }^{-1}\right)$, media $\left(3,1\right.$ a 5,0 Ton ha $\left.^{-1}\right)$, alta $(5,1$ a 10,0 Ton ha $\left.^{-1}\right)$, muy alta $\left(10,1\right.$ a 20,0 Ton $\left.^{-1} a^{-1}\right)$, severa $(20,1$ a 30 Ton $\mathrm{ha}^{-1}$ ) y muy severa $\left(>30,1\right.$ Ton ha $\left.^{-1}\right)$ (León, 2001). 
La recolección de datos se realizó en junio, julio y agosto. Esos meses han presentado precipitaciones de las más altas del año en comparación con otros periodos. Realizamos 12 lecturas de erosión por cada uno de los seis puntos para un total de 72 mediciones.

La finca cuenta con una estación meteorológica fija que cada 30 minutos proporciona datos de temperatura, precipitación, velocidad del viento, radiación solar y humedad relativa (Chamorro, 2011) de la cual se obtuvieron los datos de las precipitaciones semanales que se clasificaron en cinco grupos: débil (menor o igual a $2 \mathrm{l} / \mathrm{m}^{2}$ ), moderada (entre $2,1 \mathrm{l} / \mathrm{m}^{2}$ y $15 \mathrm{l} / \mathrm{m}^{2}$ ), fuerte (entre $15,1 \mathrm{l}$ / $\mathrm{m}^{2}$ y $30 \mathrm{l} / \mathrm{m}^{2}$ ), muy fuerte (entre $30,1 \mathrm{l} / \mathrm{m}^{2}$ y $60 \mathrm{l} / \mathrm{m}^{2}$ ) y torrencial (mayor de 60,1 l/ $\mathrm{m}^{2}$ ) (Aparicio, 2003).

Realizamos un listado taxonómico de malezas en los puntos determinados, por medio de un marco de un metro cuadrado confeccionado con tuvo de pvc de media pulgada color blanco. El marco se colocó a $9 \mathrm{~m}$ lineales del sitio de recolección de datos de erosión en espacios libres entre las matas de banano, realizando conteos taxonómicos todas las semanas en cada punto de muestreo. El marco de pvc fue señalizado con cuatro marcas de $25 \%$, cada marca se dividió en espacios de $5 \%$ y estos en otras marcas más pequeñas de $1 \%$ con las cuales se determinó el porcentaje de la presencia de las diferentes malezas o coberturas vegetales. La estimación de las malezas se realizó en forma visual colocando el marco siempre en el mismo lugar en cada sitio de muestreo y midiendo cada semana el comportamiento de las coberturas presentes y se obtuvo un inventario semanal de las malezas (Esqueda et al., 2010; Rodríguez \& Agüero, 2011).

Se analizó el costo de la mano de obra y el de los productos químicos aplicados en el periodo del muestreo para cuantificar lo gastado para combatir malezas en: a) áreas con cobertura entre el $67 \%$ y $100 \%$ de G. macropoda y b) áreas donde no se cuenta con G. macropoda. Para ambos casos se tomaron los datos correspondientes a las aplicaciones de control químico para combatir las especies vegetales no deseadas en la plantación (Muñoz, 2012).

\section{RESULTADOS}

La erosión depende de la precipitación y de la presencia de cobertura de oreja de ratón (Fig. 1). La precipitación promedio durante las 12 semanas de muestreo fue débil, de $0,21 \mathrm{~L} / \mathrm{m}^{2}\left(\max =0,71 \mathrm{~L} / \mathrm{m}^{2} ; \min =0,03\right.$; $\mathrm{DE}=0,07 ; \mathrm{n}=12$ ); en los suelos sin coberturas de $\mathrm{G}$. $m a$ cropoda la erosión aumentó con las precipitaciones (Spearman: $n=72 ; r=0,390 ; p<0,05$ ) y se obtuvo un promedio de erosión bajo, de 1,43 Ton/ha/año ( $\max =1,68$; $\min =1,30 ; D E=0,29 ; n=36$ ). En las áreas con presencia de esa cobertura la erosión no fue significativa (Spearman: $n=72 ; r=0,000 ; p<0,05)$ y la erosión promedio correspondió a $0,40 \mathrm{Ton} / \mathrm{ha} / \mathrm{año}(\max =0,48 ; \min =0,26 ; \mathrm{DE}=0,11$; $\mathrm{n}=36$ ) (Fig. 1).

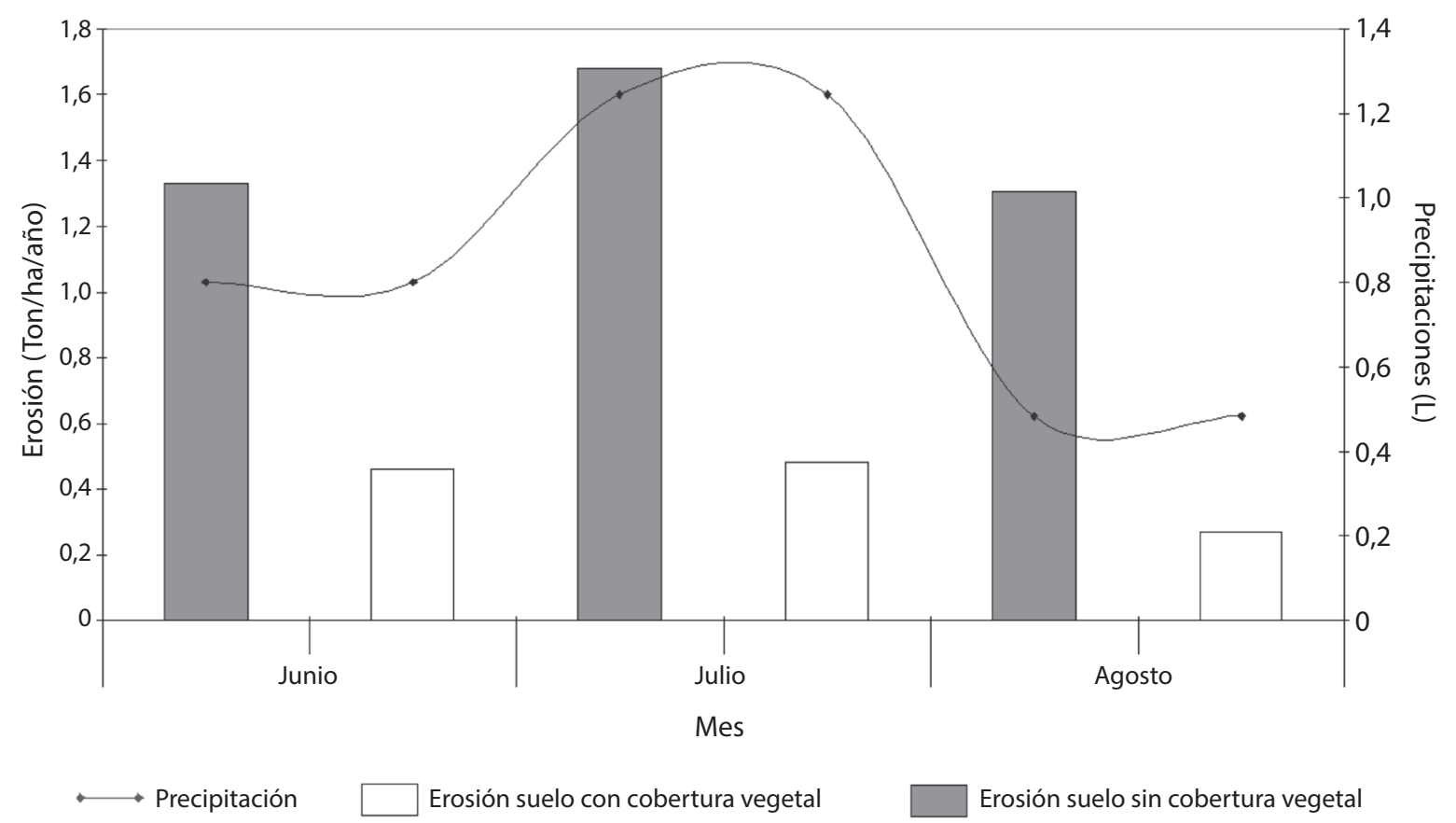

Fig. 1. Variación de erosión según la precipitación. 


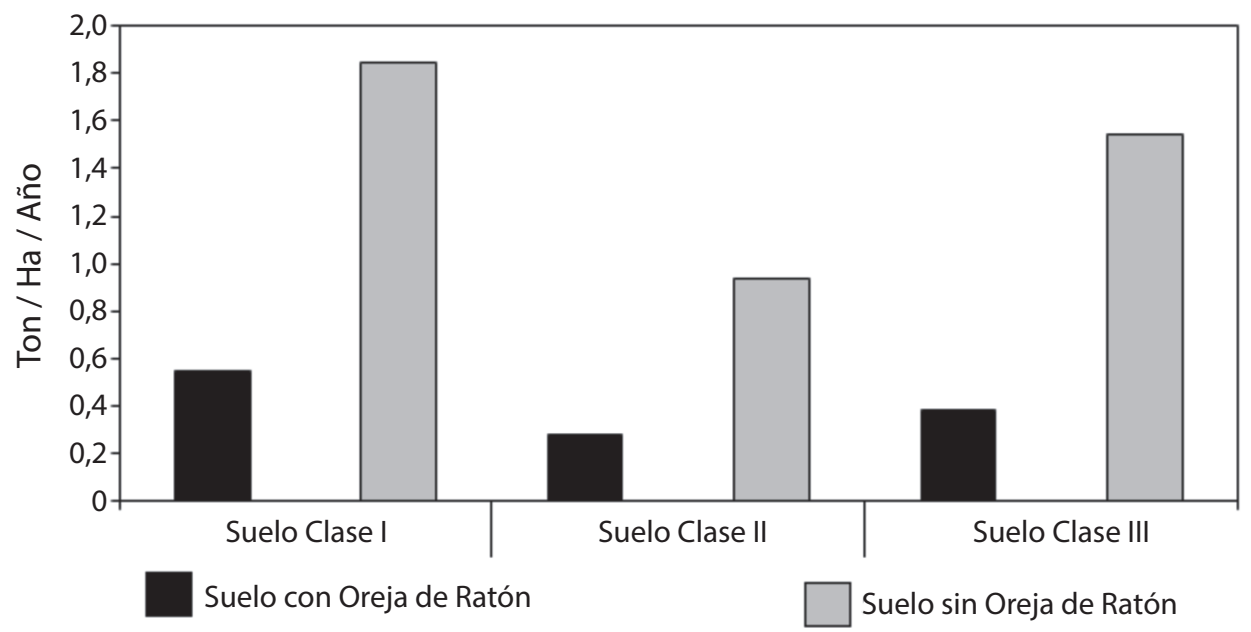

Fig. 2. Variación de erosión según la clase de suelo.

En las tres clases de suelos que contaron con cobertura de oreja de ratón se presentó menor erosión (KruskalWallis $=0,002 ; p<0,05$ ) (Fig. 2).

En un periodo de tres meses, sólo el suelo clase I (Fig. 3) requirió de una aplicación química; por lo tanto, el costo promedio por control químico de malezas en las tres clases de suelos con cobertura vegetal de G. macropoda fue de \$18 por hectárea. En los suelos sin esa cobertura vegetal el costo promedio del control de malezas en los tres tipos de suelo fue de $\$ 126$ por hectárea correspondiente a cinco aplicaciones realizadas (dos en suelo clase I, dos en clase II y una en clase III).
En el área de suelo que carecía de cobertura con oreja de ratón se encontró diversidad de malezas, pero predominó, el suelo descubierto y hojarasca con un $91 \%$ de cobertura ( $\max =98 \% ; \min =34 \% ; \mathrm{DE}=39,5 ; n=39$ ) (Fig. 4).

\section{DISCUSIÓN}

En la finca bananera observamos cambios en la cantidad de precipitación reportada para las 12 semanas de estudio, situación debida a la posición geográfica que tiene Costa Rica, en la zona tropical norte del hemisferio

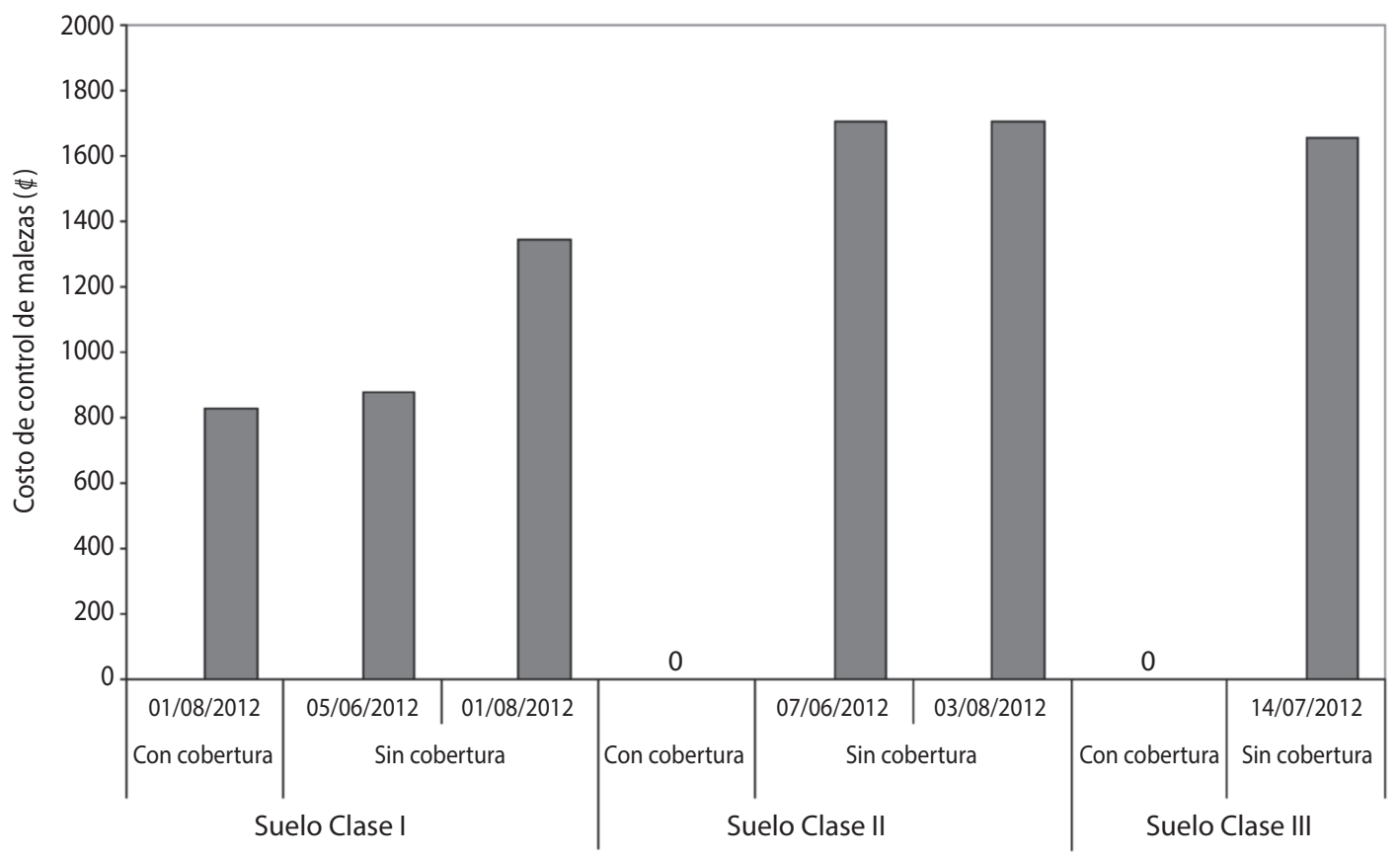

Fig. 3. Costo del control de malezas según la clase de suelo. 


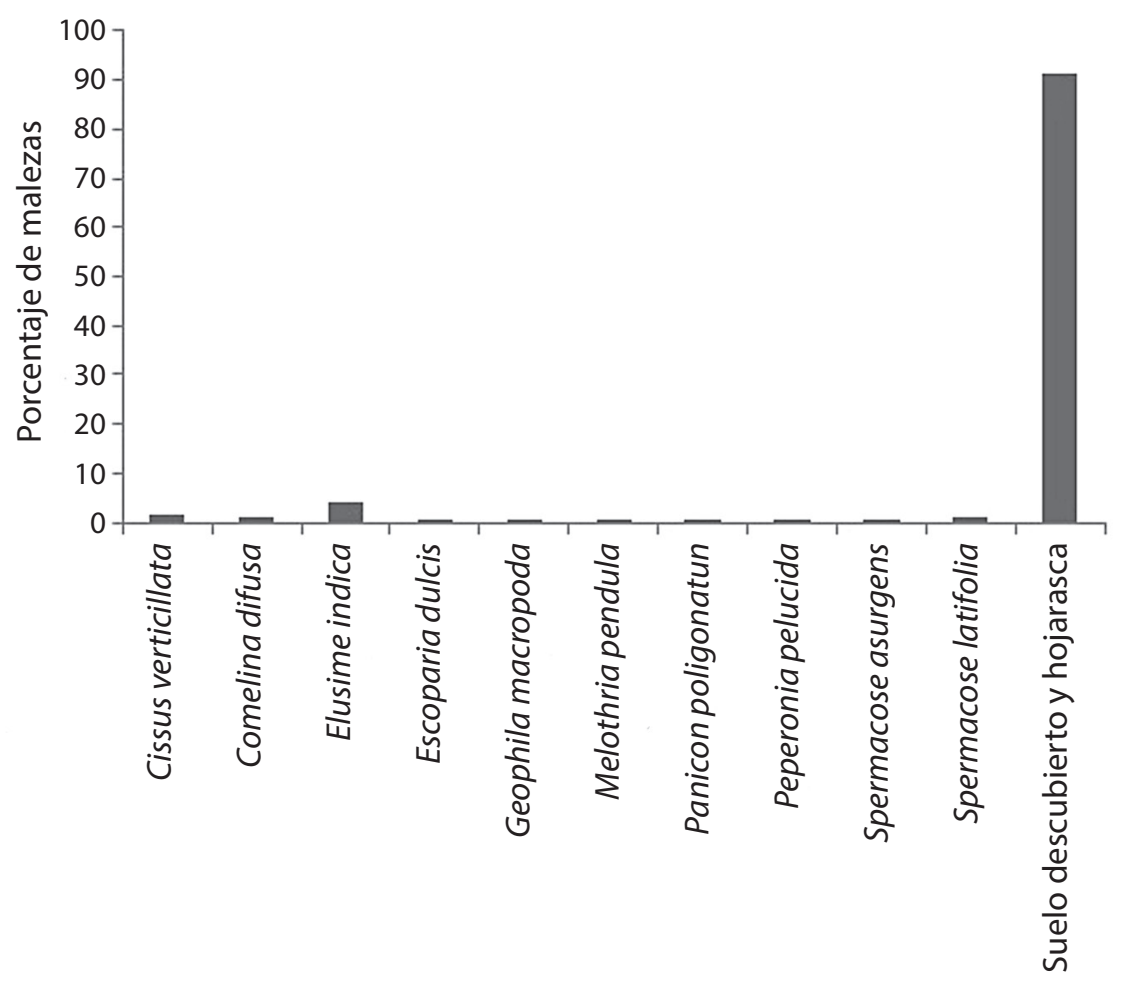

Fig. 4. Porcentaje de malezas, encontradas en áreas sin cobertura G. macropoda.

occidental, donde el clima está influenciado por los vientos Los Nortes y los Alisios, que chocan casi perpendicularmente con el sistema montañoso costarricense con dirección este a noreste (Muñoz et al., 2002).

En nuestro estudio, los suelos sin cobertura de G. macropoda presentaron dos picos de erosión, uno a inicios de junio y el otro a finales de julio, lo cual coincide con los meses de máxima precipitación para la zona (Muñoz et al., 2002). En las otras semanas de estudio, a pesar de haber existido la constante presencia de las lluvias, no se obtuvo gran cantidad de sedimento, por tratarse de lluvias de menor intensidad y cantidad. La erosión en suelos sin cobertura vegetal aumenta con las altas precipitaciones (Yglesias, 1997; Díaz et al., 1999; Arias, 2007; Brenes, 2010) y, además, se ve favorecida por las prácticas agrícolas propias del cultivo: compactación por las acciones diarias de laboreo (Alvarado et al., 2014) y ligeros movimientos de tierra [Guerrero \& Rodríguez, 2002; Ministerio de Agricultura y Ganadería (MAG), 2010].

Cada vez más se reconoce la importancia de utilizar coberturas (arvenses) en los cultivos del banano (Pinilla \& García, 2002). Se ha experimentado con G. macropoda, Desmodium ovalifolium y Pilea sp., porque tienen un crecimiento rastrero; adaptación a la sombra y al tránsito de las personas y porque aportan ventajas al cultivo del banano: eliminación de malezas, disminución en el uso de herbicidas, baja erosión, mayor biomasa por área y no afectan los porcentajes nutricionales requeridos por el cultivo (Ramos et al., 2011). También contribuyen a mejorar la infiltración del agua y la retención de humedad en el suelo; proporcionan alimento y refugio a la fauna silvestre (Labrada et al., 1996; Guerrero \& Rodríguez, 2002; Muñoz, 2012). Rescatando la erosión y el uso de $G$. macropoda como variables de nuestro estudio, se encontró que es en los suelos sin cobertura donde se presenta la mayor erosión y aumenta conforme se incrementa la precipitación (Soto, 2009; Guanca, 2010; Alvarado et al., 2014). Las coberturas con arvenses como G. macropoda, amortiguan el golpe directo de las lluvias sobre el suelo, tienen raíces que amarran más las partículas, minimizan las actividades de control químico para combatir malezas, reducen la exposición del suelo a la erosión hídrica y eólica y disminuyen las escorrentías. Por lo tanto, la práctica de suelos verdes (con cobertura) cada vez toma mayor auge entre la plantación bananera (Hudson, 1982; Morgan, 1997; Martínez, 1998; Soto, 2009; Guanca, 2010). 
Entre las prácticas del control biológico de malezas competidoras está el uso de arvenses como cobertura, uno de los recomendados es la oreja de ratón, G. macropoda (Esqueda \& Piesschaert, 1993; Araya \& Cheves, 1997; Waele et al., 2006; Vargas \& Abdelnour). Esta planta ayuda a eliminar la aparición de malezas competidoras con el cultivo del banano (Ramos et al., 2011) y protege al suelo contra la erosión, lo que reduce los costos de producción (Pinilla \& García, 2002). Si aun así, aparecen malezas en los suelos con arvenses, se controlan fácilmente con técnicas manuales de nulo impacto al ambiente, sin necesidad de aplicar sustancias químicas (Yglesias, 1997; Díaz et al., 1999; Pinilla \& García, 2002; Arias, 2007; MAG, 2008).

En las áreas de nuestro estudio que no cuentan con oreja de ratón como cobertura, existe un predominio de malezas competidoras y de hojarasca, situación que puede deberse a que en un suelo descubierto existen recursos disponibles (nutrientes, agua y espacio físico) para su desarrollo. Para su eliminación se recurrió al uso de herbicidas pues es una práctica común en dichas plantaciones (Muñoz, 2012; Ventura \& Medina, 2012).

Los suelos que cuentan con coberturas vegetales de G.F macropoda, sin importar su clase y que reciben altas precipitaciones, presentan menor grado de erosión que aquellas áreas sin esa cobertura.

\section{AGRADECIMIENTO}

A Laura Coto Vargas por acompañarme en la recolección de datos; a Zaidett Barrientos LI. y Sergio Laprade Coto por la asesoría y guía durante el desarrollo de la investigación. Rafael Segura (CORBANA) por facilitar el equipo para el secado y pesaje de las muestras de suelo; al señor Alfredo Barrantes por recoger los datos del secado de suelos. A la empresa Banacol de Costa Rica por brindar la oportunidad de desarrollar el proyecto en la finca objeto de estudio y por la colaboración en el cuidado de las áreas en prueba; a Ligia Jeannette Bermúdez por su apoyo estadístico.

\section{REFERENCIAS}

Alvarado, V., Bermudes, T., Romero, M. \& Piedra L. (2014). Plantas nativas para el control de la erosión en taludes de ríos urbanos. Spanish Journal of Soil Science. San Jose, Costa Rica.

Aparicio, J. (2003). Lluvias e inundaciones. Valencia, España. Consultado el 07 de diciembre del 2011. Disponible en: http://www.iaem.es/GuiasRiesgos/ Lluviaseinundaciones.pdf
Araya, M. \& Cheves, A. (1997). Poblaciones de los nematodos parásitos del banano (Musa aaa), en plantaciones asociadas con coberturas de Arachis pintoi y Geophilla macropoda. Agronomía Costarricense, 21(2), 217-220.

Araya, H. (2014). Inclinación del suelo de finca Teresa 05. Guápiles, Pococí, Costa Rica. Investigador de la corporación bananera nacional, conversación personal

Arias, A. (2007). Suelos tropicales. Editorial Universidad Estatal a Distancia (EUNED).San José, Costa Rica.

Brenes, S. (2010). Alternativas para el manejo de sainillo (Dieffenbachia oerstdii schott araceae) en el cultivo de banano (Musa AAA). Tesis M.sc. Ciencias agrícolas y recursos naturales con énfasis en protección de cultivos. San José, Costa Rica.

Esqueda, K. \& Piesschaert, F. (1993). Geophila macropoda (Ruiz et Pav.) DC., Prodr. 4: 537 (1830). Psychotria macropoda Ruiz et Pav., Fl. Peruv. 2: 63 (1799). Holotipo: Perú, Ruiz y Pavón s.n. (MA). Fieldiana, Bot. 33: 16.

Chacón, M. (2003). Historia y políticas nacionales de conservación. Editorial Universidad Estatal a Distancia. San José, Costa Rica.

Chamorro, J. (2011). Estadísticas de fincas al cierre año 2010. Cariari, Pococí, Costa Rica. Jefe allocation, unidad de soporte operativo, banacol de Costa Rica S.A. división banano, conversación personal

Corporación Bananera Nacional (Corbana). (2011a). Mapa interactivo. San José, Costa Rica. Consultado el 09 de agosto del 2011. Disponible en: http://www.corbana.co.cr

Díaz, J., Molina, J., Guharay, F., Zamora, M., Miranda, F. \& Zeledón R. (1999). Manejo integrado de plagas en el cultivo de repollo. Managua, Nicaragua.

Guanca, A. (2010). Determinación de la erosión hídrica de los suelos de la cuenca del río Pilcomayo - Salta. Tesis (Ing agr) Salta, Argentina.

Guerrero, M. \& Rodríguez, M. (2002). Guía técnica cultivo de plátano. La Libertad, El Salvador. Consultado el 31 de marzo del 2014.

Hudson, N. (1982). Conservación del suelo. Editorial Reverté. Barcelona, España.

Instituto Interamericano de Ciencias Agrícolas (IICA). (1989). Dinámica sobre poblaciones control de malezas en soja. Diálogo XXVI. Montevideo, Uruguay.

Kruiter, A. (1989). El banano en el norte de la zona atlántica de Costa Rica. Reporte No. 13. Turrialba, Costa Rica.

Labrada, R. Caseley, J \& Parker, C. (1996). Manejo de Malezas para Países en Desarrollo. Roma, Italia. Estudio FAO Producción y Protección Vegetal.

Laprade, S. (2012a). Conversión de gramos de suelo a toneladas. Pococí, Costa Rica. Presidente comisión ambiental bananera, conversación personal 
Laprade, S. (2012b). Estadísticas del sector bananero nacional. Pococí, Costa Rica. Presidente comisión ambiental bananera, conversación personal

León, J. (2001). Estudio y control de la erosión hídrica. Medellín, Colombia. Universidad Nacional de Colombia, Facultad de Ciencias Agropecuarias, Departamento de Ciencias Forestales.

Ministerio de Agricultura y Ganadería (MAG). (2008). Buenas prácticas agropecuarias. Ministerio de Agricultura y Ganadería. San José, Costa Rica.

Ministerio de Agricultura y Ganadería (MAG). (2010). Manual para las buenas prácticas agrícolas en la producción de piña. San José, Costa Rica.

Martínez, A. (1998). El cultivo de plátano en los llanos orientales. Villavecencia Meta, Colombia. Editorial siglo XX.

Moreno, J. Blanco, C. \& Mendoza, R. (2009). Buenas prácticas agrícolas en el cultivo de banano en la región del Magdalena. Medellín, Colombia.

Morgan, R. (1997). Erosión y conservación del suelo. Editorial Aedos. Madrid, España.

Muñoz, A.C., Fernández, W., Gutiérrez, J.A. \& Zárate, E. (2002). Variación estacional del viento en Costa Rica y su relación con los regímenes de lluvia. Instituto Tecnológico de Costa Rica, Cartago, Costa Rica. Top. Meteoro. Oceanog, 9 (1), 1-13.

Muñoz, F. (2012). Costo control de malezas en finca Teresa 05. Pococí, Costa Rica. Administrador de Compañía Bananera La Teresa S.A finca 05, conversación personal

Núñez, J. (2001). Manejo y conservación de suelos. Editorial Universidad Estatal a Distancia. San José, Costa Rica.

Pinilla, C. \& García, J. (2002). Manejo integrado de arvenses en plantaciones de banano (Musa AAA). ACROBAT. Memorias XV Reunión. Cartagena de Indias, Colombia, 222-235.

Pound, B. (2003). Cultivos de Cobertura para la Agricultura Sostenible en América Latina. Agroforestería para la Producción Animal en Latinoamérica. Roma, Italia.

Ramos, O., Vaquero, R., León, R. \& Ayuso, F. (2011). Efecto de tres especies de cobertura viva en el control de malezas en una plantación de banano orgánico (Musa aaa CV. Williams). Tierra Tropical, 7 (1), 1-10.

Reduciendo el Escurrimiento de Plaguicidas al Mar Caribe (RepCar). (2000). Informe Nacional Proyecto GEF PDF-B. San José, Costa Rica.

Rodríguez, A. \& Agüero, R. (2000). Identificación de malezas trepadoras del banano (musa sp.) en la zona caribe de Costa Rica. Agronomía Mesoamericana, 11 (1), 123-125.
Rodríguez, A. \& Agüero, R. (2011). Dinámica poblacional de panicum polygonatum y axonopus micay bajo combate con herbicidas y chapea, en una plantación bananera. Agronomía Mesoamericana, 22 (1), 117-122.

Rojas, F., Bermúdez, G. \& Jiménez, Q. (2006). Plantas ornamentales del trópico. Editorial Tecnológica de Costa Rica. Cartago, Costa Rica. 40 p.

Salguero, M. (2007). Caminos y veredas de Costa Rica. San José, Costa Rica. Universidad Estatal a Distancia.

Samuels, J. \& Segura, R. (2010). Estudio detallado de suelos de finca Teresa 05. Corporación Bananera Nacional (CORBANA). La Rita, Pococí.

Somarriba M. (2005). Manual de métodos sencillos para estimar erosión hídrica. Documento No. 592, Serie Técnica 5/2005. Managua, Nicaragua.

Soto, E. (2008). Informe anual de investigaciones 2008. Corporación Bananera Nacional (CORBANA). San José, Costa Rica.

Soto, E. (2009). Informe anual de investigaciones 2009. Corporación Bananera Nacional (CORBANA). San José, Costa Rica.

Vallejos, C. \& Velázquez, J. (1998). Evaluación de especies leguminosas como cultivos de cobertura y barreras vivas en el control de la erosión en cafetales jóvenes. Tesis (Ing Agr). Matagalpa, Nicaragua. Universidad Nacional Agraria.

Vargas, Ma. del P. \& Abdelnour, A. (2010). Cultivo in vitro de Geophila macropoda (Ruiz \& Pav. DC) a partir de embriones cigóticos. Agronomía Mesoamericana, 21 (1), 73-83.

Ventura, L. \& Medina, J. (2012). Malezas. Universidad Autónoma de Chiapas, Facultad de Ciencias Agronómicas. Chiapas, México.

Villalobos, G. (2006). Mundo laboral y vocabulario bananero en el Pacífico Sur de Costa Rica. San José, Costa Rica.

Waele, D., Stoffelen, R \& Kestemont, J. (2006). Efecto de cultivos asociados sobre nematodos Bugtok y Moko: un elemento patógeno, dos enfermedades Inoculación combinada de hongos endofíticos Variantes somaclonales Bananos ricos en micronutrientes. Revista InfoMusa, 15 (1-2), 1- 5.

Yepes, J. (2012). Beneficios para las comunidades del entorno bananero. Matina, Costa Rica. Director de Fundación Social de Banacol Costa Rica (CORBANACOL), conversación personal.

Yglesias, J. (1997). Primer taller internacional sobre control biológico y producción integrada en el cultivo de banano. Guácimo, Costa Rica. 
